\title{
Fermion Pairing Dynamics in the Relativistic Scalar Plasma
}

\author{
E. R. Takano Natti [, Chi-Yong Lin and A. F. R. de Toledo Piza \\ Instituto de Física, Universidade de São Paulo, \\ Caixa Postal 66318, CEP 05389-970, São Paulo, São Paulo, Brazil \\ P. L. Natti \\ Departamento de Matemática, Universidade Estadual de Londrina, \\ Caixa Postal 6001, Cep 86051-970, Londrina, Paraná, Brazil
}

\begin{abstract}
Using many-body techniques we obtain the time-dependent Gaussian approximation for interacting fermion-scalar field models. This method is applied to an uniform system of relativistic spin-1/2 fermion field coupled, through a Yukawa term, to a scalar field in $3+1$ dimensions, the so-called quantum scalar plasma model. The renormalization for the resulting Gaussian mean-field equations, both static and dynamical, are examined and initial conditions discussed. We also investigate solutions for the gap equation and show that the energy density has a single minimum.
\end{abstract}

Submitted to Phys. Rev. D15 (Revised Version)

PACS number(s): 11.10-z, 11.10.Lm, 11.10Gh, 21.60.Jz.

\footnotetext{
${ }^{1}$ Present address : Universidade do Norte do Paraná, Av. Paris, 675, CEP 86041-140, Londrina, Paraná, Brasil
} 


\section{Introduction}

The self-consistent mean-field method remains being one of the few analytical tools available to investigate a variety of problems in quantum field theory which cannot be handled in perturbative theory. These include static problems in the context of spontaneous symmetry breaking [1] as well as time-dependent problems involving the dynamics of the inflationdriving scalar field in the early Universe [2] and the dynamics of relaxation process in the ultra-relativistic heavy-ion experiments [3]. In its most used form the approximation is implemented, in the case of boson fields, through of a time-dependent or independent variational principle using a Gaussian trial wavefunction in the Schroedinger picture [4]. However, systematic corrections to this approximation are still an open problem. Furthermore, for the case of fermion fields, the trial wavefunction is not straightforward to take in a Gaussian form [5].

On the other hand, the mean-field description of systems of interest can be obtained from the Heisenberg equation

$$
i\langle\dot{\mathcal{O}}\rangle=\operatorname{Tr}[\mathcal{O}, H] \mathcal{F}_{0}
$$

where $\mathcal{O}$ is a one-body operator and $H$ is the Hamiltonian of the system. This can be kept under direct control when one takes a gaussian-like density. In a formulation appropriate for the many-body theory we have

$$
\mathcal{F}_{0}=\frac{\exp \left[\sum_{(i, j)} A_{i, j} \eta_{i}^{\dagger} \eta_{j}+B_{i, j} \eta_{i}^{\dagger} \eta_{j}^{\dagger}+C_{i, j} \eta_{i} \eta_{j}\right]}{\operatorname{Tr}\left\{\exp \left[\sum_{(i, j)} A_{i, j} \eta_{i}^{\dagger} \eta_{j}+B_{i, j} \eta_{i}^{\dagger} \eta_{j}^{\dagger}+C_{i, j} \eta_{i} \eta_{j}\right]\right\}}
$$

In (2) the $\eta_{i}^{\dagger}\left(\eta_{i}\right)$ are boson or fermion creation (annhihilation) operators of a particle in orbital $i$ and $\mathcal{F}_{0}$ can be, of course, rewritten in a diagonal form when one uses the Bogoliubov quasiparticle operators (cf. Eqs.(5) and (10)). It is not difficult to see that this scheme corresponds to the usual gaussian variational approximation in the functional Schroedinger picture. There are, however, some important differences. For instance, the usual technique of nonrelativistic many-fermion problem can be readily extended to the Dirac field theory [6]. 
Thus, instead of using anticommuting Grassmann variables to write Gaussian wavefunctional [5], we describe the pairing correlations by a suitable Bogoliubov unitary transformation. Furthermore, this could be the starting point for the further approximation scheme beyond the Gaussian [13]. In recent publications [8], we have applied this scheme to the $\phi^{4}$ theory and Chiral Gross-Neveu Model (hereafter referred to as I,II).

The main point of this paper is to apply this method within the context of interacting fermion-boson models. As a first step towards this end, Takano Natti and de Toledo Piza 99 have obtained relevant dynamics for the Jaynes-Cummings Hamiltonian, a well known model in quantum optics [10]. This can be seen as $0+1$ dimensional quantum field theory known as relativistic scalar plasma [11]. Exact numerical results have been useful in this case to assess the quality of various approximation schemes. In particular, we have shown that the method described here is a good approximation whenever a moving Gaussian has enough freedom to mimic the complicated evolution of the exact wave function. In this paper, we report an aplication of the same technique to describe the real-time evolution for a fermion field interacting with scalar boson in $3+1$ dimensions. An outline of the paper is as follows. In Sec. II we extend the discussions of I and II to a system countaing interacting boson and fermion fields. Sec. III will illustre this scheme in the simplest context of scalar plasma model. In Sec. IV we discuss the self-consistent renormalization for the resulting equations in the equilibrium situation. The renormalization of the time-dependent equations is then considered in Sec V and finite initial conditions discussed.

\section{Kinetic of Interacting Fermion-Scalar Systems}

The present discussion for the real-time evolution of theories containing both scalar and fermion fields basically follows the earlier works in the context of $\phi^{4}$ and Chiral-Gross-Neveu models. The basic idea of our approach is to focus on the time evolution of the expectation values of linear, $\phi(x)$, and bilinear field operators such as $\phi(x) \phi(x), \bar{\psi}(x) \psi(x), \psi(x) \psi(x)$ and etc, referred to as Gaussian observables. Many technical details of the method can be found 
in I and II, and we restrict ourselves here to a few key steps of the derivation of equations of motion for the present case.

Let us consider first the bosonic sector of the system. The Heisenberg field operators $\phi(x)$ and $\Pi(x)$ are expanded as

$$
\begin{aligned}
\phi(\mathbf{x}, t) & =\sum_{\mathbf{p}} \frac{1}{\left(2 V p_{0}\right)^{1 / 2}}\left[b_{\mathbf{p}}(t) e^{i \mathbf{p} \cdot \mathbf{x}}+b_{\mathbf{p}}^{\dagger}(t) e^{-i \mathbf{p} \cdot \mathbf{x}}\right] \\
\Pi(\mathbf{x}, t) & =i \sum_{\mathbf{p}}\left(\frac{V p_{0}}{2}\right)^{1 / 2}\left[b_{\mathbf{p}}^{\dagger}(t) e^{-i \mathbf{p} \cdot \mathbf{x}}-b_{\mathbf{p}}(t) e^{i \mathbf{p} \cdot \mathbf{x}}\right],
\end{aligned}
$$

where $b_{\mathbf{p}}^{\dagger}(t)$ and $b_{\mathbf{p}}(t)$ are the usual boson creation and annihilation operators satisfying the standard commutation relations at equal times

$$
\left[b_{\mathbf{p}}(t), b_{\mathbf{p}^{\prime}}^{\dagger}\left(t^{\prime}\right)\right]_{t=t^{\prime}}=\delta_{\mathbf{p}, \mathbf{p}^{\prime}}, \quad\left[b_{\mathbf{p}}^{\dagger}(t), b_{\mathbf{p}^{\prime}}^{\dagger}\left(t^{\prime}\right)\right]_{t=t^{\prime}}=\left[b_{\mathbf{p}}(t), b_{\mathbf{p}^{\prime}}\left(t^{\prime}\right)\right]_{t=t^{\prime}}=0
$$

In Eq.(3) $V$ is the volume of the periodicity box,

$$
\left(p_{0}\right)^{2}=\mathbf{p}^{2}+\Omega^{2} \quad \text { and } \quad p x=p_{0} t-\mathbf{p} \cdot \mathbf{x}
$$

where $\Omega$ is the mass parameter and will be fixed later.

In order to deal with condensate and pairing dynamics of the scalar field we follow Sec. II of I and define a unitary Bogoliubov transformation as

$$
\left[\begin{array}{c}
\beta_{\mathbf{p}} \\
\beta_{-\mathbf{p}}^{\dagger}
\end{array}\right]=\left[\begin{array}{cc}
\cosh \kappa_{\mathrm{p}}-i \frac{\eta_{\mathrm{p}}}{2} & \sinh \kappa_{\mathrm{p}}-i \frac{\eta_{\mathrm{p}}}{2} \\
\sinh \kappa_{\mathrm{p}}+i \frac{\eta_{\mathrm{p}}}{2} & \cosh \kappa_{\mathrm{p}}+i \frac{\eta_{\mathrm{p}}}{2}
\end{array}\right]\left[\begin{array}{c}
d_{\mathbf{p}} \\
d_{-\mathbf{p}}^{\dagger}
\end{array}\right],
$$

where $d_{\mathbf{p}}$ is the shifted boson operator

$$
d_{\mathbf{p}}(t) \equiv b_{\mathbf{p}}(t)-B(t) \delta_{\mathbf{p}, 0} \quad \text { with } \quad B_{\mathbf{p}}(t) \equiv\left\langle b_{\mathbf{p}}(t)\right\rangle=\operatorname{Tr}_{\mathrm{BF}}\left[b_{\mathbf{p}}(t) \mathcal{F}_{1}\right]
$$

Here and in what follows the symbol $\operatorname{Tr}_{\mathrm{BF}}$ denotes a trace over both bosonic and fermionic variables. Partial traces over bosonic or fermionic variables will be written as $T r_{\mathrm{B}}$ and $\operatorname{Tr}_{\mathrm{F}}$ respectively. The Bogoliubov coefficients are determined from the secular problem given in 
(2.8)-(2.10) of I to yield $\left\langle\beta_{\mathbf{p}} \beta_{-\mathbf{p}}\right\rangle=0$. The resulting eigenvalues, $\nu_{\mathbf{p}}=\left\langle\beta_{\mathbf{p}}^{\dagger} \beta_{\mathbf{p}}\right\rangle$, are quasiparticle occupation numbers.

For the fermion field, we follow the discussion of II and decompose the field operators in Fourier components,

$$
\begin{aligned}
& \psi(\mathbf{x}, t)=\sum_{\mathbf{k}, s}\left(\frac{M}{k_{0}}\right)^{1 / 2} \frac{1}{\sqrt{V}}\left[u_{1}(\mathbf{k}, s) a_{\mathbf{k}, s}^{(1)}(t) e^{i \mathbf{k} \cdot \mathbf{x}}+u_{2}(\mathbf{k}, s) a_{\mathbf{k}, s}^{(2) \dagger}(t) e^{-i \mathbf{k} \cdot \mathbf{x}}\right] \\
& \bar{\psi}(\mathbf{x}, t)=\sum_{\mathbf{k}, s}\left(\frac{M}{k_{0}}\right)^{1 / 2} \frac{1}{\sqrt{V}}\left[\bar{u}_{1}(\mathbf{k}, s) a_{\mathbf{k}, s}^{(1)^{\dagger}}(t) e^{-i \mathbf{k} \cdot \mathbf{x}}+\bar{u}_{2}(\mathbf{k}, s) a_{\mathbf{k}, s}^{(2)}(t) e^{i \mathbf{k} \cdot \mathbf{x}}\right]
\end{aligned}
$$

where $a_{\mathbf{k}, s}^{(1)}{ }^{\dagger}(t)$ and $a_{\mathbf{k}, s}^{(1)}(t)\left[a_{\mathbf{k}, s}^{(2)^{\dagger}}(t)\right.$ and $\left.a_{\mathbf{k}, s}^{(2)}(t)\right]$ are fermion creation and annihilation operators associated with positive [negative]-energy solutions $u_{1}(\mathbf{k}, s)\left[u_{2}(\mathbf{k}, s)\right]$ and satisfy the anticommutation rules

$$
\begin{aligned}
\left\{a_{\mathbf{k}, s}^{(\lambda)^{\dagger}}(t), a_{\mathbf{k}^{\prime}, s^{\prime}}^{\left(\lambda^{\prime}\right)}\left(t^{\prime}\right)\right\}_{t=t^{\prime}} & =\delta_{\mathbf{k}, \mathbf{k}^{\prime}} \delta_{s, s^{\prime}} \delta_{\lambda, \lambda^{\prime}} \quad \text { for } \quad \lambda, \lambda^{\prime}=1,2 \\
\left\{a_{\mathbf{k}, s}^{(\lambda)^{\dagger}}(t), a_{\mathbf{k}^{\prime}, s^{\prime}}^{\left(\lambda^{\prime}\right)}\left(t^{\prime}\right)\right\}_{t=t^{\prime}} & =\left\{a_{\mathbf{k}, s}^{(\lambda)}(t), a_{\mathbf{k}^{\prime}, s^{\prime}}^{\left(\lambda^{\prime}\right)}\left(t^{\prime}\right)\right\}_{t=t^{\prime}}=0 .
\end{aligned}
$$

In Eq.(17) the spinors are normalized to $k_{0} / M$ and

$$
\left(k_{0}\right)^{2}=\mathbf{k}^{2}+M^{2} \quad \text { and } \quad k x=k_{0} t-\mathbf{k} \cdot \mathbf{x}
$$

where $M$ is a mass parameter for the fermions.

Adapting Eqs. (3)-(11) and (30) of II to the present case of $3+1$ dimensional field we can write the Bogoliubov quasi-particle operators as

$$
\left[\begin{array}{c}
\alpha_{\mathbf{k}, s}^{(1)} \\
\alpha_{\mathbf{k}, s}^{(2)} \\
\alpha_{-\mathbf{k}, \bar{s}}^{(1) \dagger} \\
\alpha_{-\mathbf{k}, \bar{s}}^{(2)}{ }_{\dagger}^{\dagger}
\end{array}\right]=\left[\begin{array}{cccc}
\cos \varphi_{\mathbf{k}} & 0 & 0 & e^{-i \gamma_{\mathbf{k}}} \sin \varphi_{\mathbf{k}} \\
0 & \cos \varphi_{\mathbf{k}} & -e^{-i \gamma_{\mathbf{k}}} \sin \varphi_{\mathbf{k}} & 0 \\
0 & e^{i \gamma_{\mathbf{k}}} \sin \varphi_{\mathbf{k}} & \cos \varphi_{\mathbf{k}} & 0 \\
-e^{i \gamma_{\mathbf{k}}} \sin \varphi_{\mathbf{k}} & 0 & 0 & \cos \varphi_{\mathbf{k}}
\end{array}\right]\left[\begin{array}{c}
a_{\mathbf{k}, s}^{(1)} \\
a_{\mathbf{k}, s}^{(2)} \\
a_{-\mathbf{k}, \bar{s}}^{(1)} \\
a_{-\mathbf{k}, \bar{s}}^{(2)}{ }^{\dagger}
\end{array}\right]
$$


and require $\left\langle\alpha_{-\mathbf{k}, \bar{s}}^{\left(\lambda^{\prime}\right)} \alpha_{\mathbf{k}, s}^{(\lambda)}\right\rangle=0$ in order to determine this unitary transformation. The quasifermion occupation number will be given by $\nu_{\mathbf{k}, s}^{(\lambda)}=\left\langle\alpha_{\mathbf{k}, s}^{(\lambda)^{\dagger}} \alpha_{\mathbf{k}, s}^{(\lambda)}\right\rangle$. In (10) we used $|\bar{s}\rangle=$ $(-)^{1 / 2-s}|-s\rangle$ to write the spin labels.

The next step is to obtain the equations of motion for the condensate, $\langle\phi\rangle$ and $\langle\Pi\rangle$, and for the Bogoliubov parameters given in (5)-(6) and (10). This proceeds by using the Heisenberg equation of motion (11), where $\mathcal{O}$ are performed by linear or bilinear combinations of creation and annihilation parts of the field operators [see (2.17) of I and (5) of II for details]. For the bosonic variables we have

$$
\begin{aligned}
i\langle\dot{\phi}\rangle & =\operatorname{Tr}_{\mathrm{BF}}[\phi, H] \mathcal{F}_{0} \\
i\langle\dot{\Pi}\rangle & =\operatorname{Tr}_{\mathrm{BF}}[\Pi, H] \mathcal{F}_{0} \\
\dot{\nu}_{\mathrm{p}} & =\operatorname{Tr}_{\mathrm{BF}}\left[\beta_{\mathbf{p}}^{\dagger} \beta_{\mathbf{p}}, H\right] \mathcal{F}_{0} \\
-i \dot{\kappa}_{\mathrm{p}}-e^{-\kappa_{\mathrm{p}}}\left(\dot{\eta}_{\mathrm{p}}+\dot{\kappa}_{\mathrm{p}} \eta_{\mathrm{p}}\right) & =\frac{\operatorname{Tr}\left[\beta_{\mathbf{p}}^{\dagger} \beta_{-\mathbf{p}}^{\dagger}, H\right] \mathcal{F}_{0}}{1+2 \nu_{\mathrm{p}}} .
\end{aligned}
$$

Analogously, the dynamical equations for fermionic variables read as

$$
\begin{aligned}
\dot{\nu}_{\mathbf{k}, s}^{(\lambda)} & =\operatorname{Tr}_{\mathrm{BF}}\left(\left[\alpha_{\mathbf{k}, s}^{(\lambda)^{\dagger}} \alpha_{\mathbf{k}, s}^{(\lambda)}, H\right] \mathcal{F}_{0}\right) \\
{\left[i \dot{\varphi}_{\mathbf{k}}+\dot{\gamma}_{\mathbf{k}} \sin \varphi_{\mathbf{k}} \cos \varphi_{\mathbf{k}}\right] e^{-i \gamma_{\mathbf{k}}} } & =\frac{\operatorname{Tr}_{\mathrm{BF}}\left(\left[\alpha_{-\mathbf{k}, s}^{(1)} \alpha_{\mathbf{k}, s}^{(2)}, H\right] \mathcal{F}_{0}\right)}{1-\nu_{\mathrm{k}, s}^{(1)}-\nu_{\mathbf{k}, s}^{(2)}}
\end{aligned}
$$

Our implementation of the Gaussian mean-field approximation consists in assuming a factorized form for the density matrix [9]

$$
\mathcal{F}_{0}(t)=\mathcal{F}_{0}^{\mathrm{B}} \mathcal{F}_{0}^{\mathrm{F}}
$$

In Eq.(17) the subsystem densities $\mathcal{F}_{0}^{\mathrm{B}}$ and $\mathcal{F}_{0}^{\mathrm{F}}$ are gaussian densities given in Eq.(2), now 
rewritten in the diagonal forms using the Bogoliubov quasiboson and quasifermion operators,

$$
\begin{gathered}
\mathcal{F}_{0}^{\mathrm{B}}=\prod_{\mathbf{p}} \frac{1}{1+\nu_{\mathbf{p}}}\left(\frac{\nu_{\mathbf{p}}}{1+\nu_{\mathbf{p}}}\right)^{\beta_{\mathbf{p}}^{\dagger} \beta_{\mathbf{p}}}, \\
\mathcal{F}_{0}^{\mathrm{F}}=\prod_{\mathbf{k}, s, \lambda}\left[\nu_{\mathbf{k}, s}^{(\lambda)} \alpha_{\mathbf{k}, s}^{(\lambda)^{\dagger}} \alpha_{\mathbf{k}, s}^{(\lambda)}+\left(1-\nu_{\mathbf{k}, s}^{(\lambda)}\right) \alpha_{\mathbf{k}, s}^{(\lambda)} \alpha_{\mathbf{k}, s}^{(\lambda)^{\dagger}}\right] .
\end{gathered}
$$

Substituting $\mathcal{F}_{0}$ into (13) and (15) one verifies immediately that the occupation numbers are constant,

$$
\dot{\nu}_{\mathrm{p}}=\operatorname{Tr}_{\mathrm{BF}}\left[\beta_{\mathbf{p}}^{\dagger} \beta_{\mathbf{p}}, H\right] \mathcal{F}_{0}=\dot{\nu}_{\mathbf{k}, \lambda}=\operatorname{Tr}_{\mathrm{BF}}\left[\alpha_{\mathbf{k}, s}^{(\lambda)^{\dagger}} \alpha_{\mathbf{k}, s}^{(\lambda)}, H\right] \mathcal{F}_{0}=0
$$

Thus, Eqs.(11)-(16) form a set of self-consistent equations of motion to the Gaussian variables. Its implementation for a specific model consists essentially in taking the traces of appropriate commutators in the Fock space. The fact that occupancies are constant implies that the entropy function associated with $\mathcal{F}_{0}$, i.e., $S=-\operatorname{Tr}_{\mathrm{BF}} \mathcal{F}_{0} \log \mathcal{F}_{0}$, does not change in time. One recovers therefore the usual isoentropic character of the Gaussian approximation.

A method to improve the mean-field approximation in field theory was discussed in I. The approach follows the line of thinking of a time-dependent projection technique proposed some time ago by Willis and Picard [12] in the context of master equation for coupled systems and extended later by Nemes and de Toledo Piza to study nonrelativistic many-fermion dynamics [13]. The method consists essentially in writting the correlation information of the full density of the system in terms of a memory kernel acting on the uncorrelated density $F_{0}$, with the help of a time-dependent projector (see Sec. III and Appendix A of I for details). At this point, an systematic mean-field expansion for two-point correlations can be perfomed. The lowest order reported in this work corresponds to the results of the usual Gaussian approximation. The higher orders describe the dynamical correlation effects between the subsystems and are expressed through suitable memorial integrals added to the mean-field dynamical equations. Thus, the resulting equations acquire the structure of kinetic equations, with the memory integrals performing as collisional dynamics terms which eliminate the isoentropic constraint. 
This higher order approximation has been implemented for the case of $0+1$ dimensional field model [9] and will not be done in this paper.

\section{Effective Dynamics of Relativistic Scalar Plasma}

The procedure discussed in the preceding section is quite general and can be, in principle, utilized for systems containing scalar and spin-1/2 particles. This is not, however a straightforward task, since divergences appear in the equations require a discussion of the renormalizability of specific models of interest. In Ref. [14 properties and stability of possible vacuum states for several fermion-scalar models utilizing the Gaussian-effective-potential (GEP) approach have been studied. It is shown that in $3+1$ dimensions the models seem doomed to instability and the GEP is bounded below only if the Yukawa coupling vanishes. We have reached a similar conclusion in an early stage of this work.

In order to give a well defined physical application, we shall adopt here the relativistic scalar plasma model widely used within the context of ultrahigh dense matter [15]. This system consists of relativistic fermion gas interacting through a massive scalar meson only and corresponds to one of the simplest quantum-field theoretical models used to discuss the relativistic dense matter in the contexts of heavy-ion collisions and the high-density astrophysical system [11]. In this application, one considers the mean-field contributions, $\langle\phi\rangle$, are much larger than the flutuactions, $\left\langle\phi^{2}\right\rangle-\langle\phi\rangle^{2}$. Therefore, the bosonic pairing is ignored and Bogoliubov transformation (5) reduces to identity. We shall show in the next sections that the resulting equations of interest in this case can be made finite by introducing appropriate counterterms.

We now proceed to apply the technique discussed in Sec. II to the scalar plasma model. The dynamics is governed by the Hamiltonian (we use the notation: $\int_{\mathbf{x}} \equiv \int d^{3} x$ )

$$
\begin{aligned}
H & =\int_{\mathbf{x}} \mathcal{H}, \\
\mathcal{H} & =-\bar{\psi}(i \vec{\gamma} \cdot \vec{\partial}-m) \psi-g \bar{\psi} \phi \psi+\frac{1}{8 \pi}\left[(4 \pi \Pi)^{2}+|\partial \phi|^{2}+\mu^{2} \phi^{2}\right]+\mathcal{H}_{c},
\end{aligned}
$$


where the parameters $m$ and $\mu$ are, respectively, the mass of fermion and scalar particles and $g$ is the coupling constant. The last term of this expression contains the counterterms necessary to remove the infinities occurring later [15],

$$
4 \pi \mathcal{H}_{\mathrm{C}}=\frac{A}{1 !} \phi+\frac{\delta \mu^{2}}{2 !} \phi^{2}+\frac{C}{3 !} \phi^{3}+\frac{D}{4 !} \phi^{4}
$$

where the coeffcients $A, \delta \mu^{2}, C$ and $D$ are infinite constants to be defined later.

Introducing these ingredients into Eqs.(11), (12) and (16) one easily obtains dynamical equation for the condensate

$$
\begin{aligned}
\langle\dot{\phi}\rangle & =4 \pi\langle\Pi\rangle \\
\langle\dot{\Pi}\rangle & =-\frac{1}{4 \pi}\left[A+\frac{C}{2} G(\Omega)\right]-\frac{1}{4 \pi}\left[\mu^{2}+\delta \mu^{2}+\frac{D}{2} G(\Omega)\right]\langle\phi\rangle-\frac{C}{8 \pi}\langle\phi\rangle^{2}-\frac{D}{24 \pi}\langle\phi\rangle^{3} \\
& -g \sum_{s} \int_{\mathrm{k}} \frac{1}{k_{0}}\left[M \cos 2 \varphi_{\mathrm{k}}+k \sin 2 \varphi_{\mathrm{k}} \cos \gamma_{\mathrm{k}}\right]\left(1-\nu_{\mathrm{k}, s}^{(1)}-\nu_{\mathrm{k}, s}^{(2)}\right),
\end{aligned}
$$

where $k \equiv|\mathbf{k}|$, and two real equations for the fermion pairing

$$
\begin{aligned}
\dot{\varphi}_{\mathrm{k}} & =\frac{k}{k_{0}}(M-\bar{m}) \sin \gamma_{\mathrm{k}} \\
\sin 2 \varphi_{\mathrm{k}} \dot{\gamma}_{\mathrm{k}} & =\frac{2\left(\mathbf{k}^{2}+\bar{m} M\right)}{k_{0}} \sin 2 \varphi_{\mathrm{k}}+\frac{2(M-\bar{m})}{k_{0}} k \cos 2 \varphi_{\mathrm{k}} \cos \gamma_{\mathrm{k}}
\end{aligned}
$$

In (24) we have introduced the notation

$$
G(\Omega)=\int_{\mathbf{p}} \frac{1+2 \nu_{\mathbf{p}}}{2 \sqrt{\mathbf{p}^{2}+\Omega^{2}}}=\frac{1}{8 \pi^{2}}\left(\Lambda_{\mathbf{p}}^{2}+\Omega^{2} \log \frac{2 \Lambda_{\mathbf{p}}}{\sqrt{e} \Omega}\right)+\int_{\mathbf{p}} \frac{\nu_{\mathbf{p}}}{p_{0}}
$$

where $\Lambda_{\mathbf{p}}$ is a momentum cutoff used in evaluating the integral. In (25)-(26) $\bar{m} \equiv m-g\langle\phi\rangle$ stands for the effective fermion mass. 
Another physical quantity of interest is the energy density of the system,

$$
\begin{aligned}
\frac{\langle H\rangle}{V}= & \frac{1}{V} \operatorname{Tr} H \mathcal{F}_{0} \\
= & -\sum_{s} \int_{\mathrm{k}}\left[\frac{\left(\mathbf{k}^{2}+\bar{m} M\right)}{k_{0}} \cos 2 \varphi_{\mathrm{k}}+\frac{(\bar{m}-M)}{k_{0}} k \sin 2 \varphi_{\mathrm{k}} \cos \gamma_{\mathrm{k}}\right]\left(1-\nu_{\mathrm{k}}^{(1)}-\nu_{\mathrm{k}}^{(2)}\right) \\
& +\frac{1}{8 \pi}\left[\langle\Pi\rangle^{2}+\mu^{2}\langle\phi\rangle^{2}\right]+\frac{1}{4 \pi}\left[A+\frac{C}{2} G(\Omega)\right]\langle\phi\rangle+\frac{1}{8 \pi}\left[\delta \mu^{2}+\frac{D}{2} G(\Omega)\right]\langle\phi\rangle^{2} \\
& +\frac{C}{24 \pi}\langle\phi\rangle^{3}+\frac{D}{96 \pi}\langle\phi\rangle^{4}+\frac{1}{8 \pi}\left[\mu^{2}+\delta \mu^{2}\right] G(\Omega)+\frac{D}{32 \pi} G^{2}(\Omega) .
\end{aligned}
$$

An important feature of this scheme is that the mean energy is conserved, a property which can be verified explicitly using the equations of motion (23) $-(26)$. Notice also that the results above contain divergent integrals, so that the coefficients $A, \delta \mu^{2}, C$ and $D$ have to be adjusted appropriately in order to give a well defined dynamics. We shall focus on this point in the next sections.

\section{IV-Static Equations and Renormalization}

This section considers Eqs. (23)-(26) in the equilibrium situation. We shall investigate the solution of these equations and study renormalization conditions. Hence, we set

$$
\dot{\gamma}_{\mathrm{k}}=\dot{\varphi}_{\mathrm{k}}=\langle\dot{\phi}\rangle=\langle\dot{\Pi}\rangle=0
$$

Since our main goal here is to set up a Gaussian mean field approximation for interacting fermion-boson fields, we shall, for simplicity, not consider the matter contribution in the present calculation. This can be done in the case of Gaussian mean-field approximation by assuming an appropriate statistical distribution to the occupation numbers $\nu_{\mathrm{p}}$ and $\nu_{\mathrm{k}, \mathrm{s}}^{(\lambda)}$ as initial conditions in (13) and (15). Thus, for the rest of discussion we have vanishing occupancies, $\nu_{\mathrm{p}}=\nu_{\mathrm{k}, \mathrm{s}}^{(\lambda)}=0$, and the sum in spin gives a numerical factor, $\sum_{s}=2$. With these assumptions, Eqs.(23)-(26) become 


$$
\begin{aligned}
& \left.\langle\Pi\rangle\right|_{\mathrm{eq}}=0 \\
& {\left[A+\frac{C}{2} G(\Omega)\right]+\left.\left[\mu^{2}+\delta \mu^{2}+\frac{D}{2} G(\Omega)\right]\langle\phi\rangle\right|_{\mathrm{eq}}+\left.\frac{C}{2}\langle\phi\rangle\right|_{\mathrm{eq}} ^{2}+\left.\frac{D}{6}\langle\phi\rangle\right|_{\mathrm{eq}} ^{3}} \\
& +8 \pi g \int_{\mathrm{k}} \frac{1}{k_{0}}\left(\left.M \cos 2 \varphi_{\mathrm{k}}\right|_{\mathrm{eq}}+\left.\left.k \sin 2 \varphi_{\mathrm{k}}\right|_{\mathrm{eq}} \cos \gamma_{\mathrm{k}}\right|_{\mathrm{eq}}\right)=0 \\
& \left.(M-\bar{m}) \sin \gamma_{\mathrm{k}}\right|_{\mathrm{eq}}=0 \\
& \left.\left(\mathbf{k}^{2}+\bar{m} M\right) \sin 2 \varphi_{\mathrm{k}}\right|_{\mathrm{eq}}+\left.\left.(M-\bar{m}) \cos 2 \varphi_{\mathrm{k}}\right|_{\mathrm{eq}} \cos \gamma_{\mathrm{k}}\right|_{\mathrm{eq}}=0
\end{aligned}
$$

where $\bar{m}$ is now the effective fermion mass calculated at $\left.\langle\phi\rangle\right|_{\text {eq }}$. Solutions of this set of equations correspond to a stationary points of the energy density, and include the ground state of the system in this approximation.

\section{A. Equilibrium conditions and mass parameters}

Considering equations (32) and (33) one sees that two situations have to be analysed.

i) $\left.\sin \gamma_{\mathrm{k}}\right|_{\text {eq }}=0$

Using this solution in Eq.(33) we have

$$
\left.\cos 2 \varphi_{\mathrm{k}}\right|_{\mathrm{eq}}= \pm \frac{\left(\mathbf{k}^{2}+\bar{m} M\right)}{k_{0} \bar{k}_{0}},\left.\quad \sin 2 \varphi_{\mathrm{k}}\right|_{\mathrm{eq}}=\mp \frac{M-\bar{m}}{k_{0} \bar{k}_{0}}
$$

where $\bar{k}_{0}=\sqrt{\mathbf{k}^{2}+\bar{m}^{2}}$. Substituting this result into (31) one finds for this case the following gap equation

$$
\left[A+\frac{C}{2} G(\Omega)\right]+\left.\left[\mu^{2}+\delta \mu^{2}+\frac{D}{2} G(\Omega)\right]\langle\phi\rangle\right|_{\mathrm{eq}}+\left.\frac{C}{2}\langle\phi\rangle\right|_{\mathrm{eq}} ^{2}+\left.\frac{D}{6}\langle\phi\rangle\right|_{\mathrm{eq}} ^{3}= \pm 16 \pi g \bar{m} G(\bar{m}),
$$

where $G(\bar{m})$ is the divergent integral defined in (27) with $\nu_{\mathrm{p}}=0$. 
ii) $M=\bar{m}$

In this case $\gamma_{\mathrm{k}}$ can have any value and (33) has as solution

$$
\left.\sin 2 \varphi_{\mathrm{k}}\right|_{\mathrm{eq}}=0 \quad,\left.\quad \cos 2 \varphi_{\mathrm{k}}\right|_{\mathrm{eq}}= \pm 1
$$

Comparing now (34) with (36) one notices immediately that the former includes the second solution as a particular case, where the mass parameter $M$ is defined here by the equilibrium condition and Bogoliubov transformation (10) reduces to an identity matrix.

The above discussion shows that the equilibrium value $\left.\langle\phi\rangle\right|_{\text {eq }}$ of the condensate, or the effective fermion mass $\bar{m}$ are independent of the initial choice of the mass paramete $M$. In fact, the effects of pairing transformation will be such that the effective fermion mass is always given by the equilibrium value calculated with (35). (See also (43) below for the mean-field energy). Henceforth, we shall thus take $M=m$ without loss of generality.

\section{B. Counterterms}

The arbitrary parameters $A, \delta \mu, C$ and $D$ can be fixed easily by adjusting the coefficients of $\left.\langle\phi\rangle\right|_{\text {eq }}$ in both of sides of (35) to give a finite gap equation. Thus, taking account the \pm signs in (35) we choose the following self-consistent renormalization prescription

$$
\begin{aligned}
D & = \pm 48 \pi g^{4} L(m) \\
\delta \mu^{2} & =\mp 24 \pi^{2} g^{4} L(m) G(\mu) \mp 16 \pi g^{2} G(0) \pm 24 \pi m^{2} g^{2} L(m) \\
C & =\mp 48 \pi m g^{3} L(m) \\
A & = \pm 24 \pi m g^{3} L(m) G(\mu) \pm 16 \pi m g G(m),
\end{aligned}
$$

where

$$
L(m) \equiv \int_{\mathbf{k}} \frac{1}{2 \mathbf{k}^{2}\left(\mathbf{k}^{2}+m^{2}\right)^{1 / 2}}=\frac{1}{4 \pi^{2}} \log \frac{2 \Lambda_{\mathbf{p}}}{m} .
$$

Furthermore, $\mu$ and $m$ are the mass scales for boson and fermion fields respectively. 
Substitution of these counterterms into (35) produce the appropriate cancelations which render the equation finite. A combination of type $L(m)[G(\mu)-G(\Omega)]$ comes from the first two terms. Since $\Omega$ is an arbitrary expansion mass parameter, one can remove this divergence by setting $\Omega=\mu$. The resulting finite gap equation is

$$
\left.\frac{\pi}{2} \mu^{2}\langle\phi\rangle\right|_{\mathrm{eq}}-g \bar{m}^{3}\left[\ln \left(\frac{\bar{m}}{m}\right)+\frac{1}{2}\right]=0
$$

The above equation together with (32)-(33) determine the stationary points of the model in the Gaussian mean-field approximation.

\section{Mean energy and stationary solutions}

We examine next the energy density when it is stationary with respect to the fermion variables. With the help of (34), Eq.(28) becomes

$$
\begin{aligned}
\frac{\langle H\rangle}{V}\left(\left.\varphi_{\mathrm{k}}\right|_{\mathrm{eq}},\left.\gamma_{\mathrm{k}}\right|_{\mathrm{eq}},\langle\phi\rangle\right) & =\mp \int_{\mathrm{k}} G^{-1}(\bar{m})+\frac{1}{4 \pi}\left[A+\frac{C}{2} G(\mu)\right]\langle\phi\rangle+\frac{1}{8 \pi}\left[\mu^{2}+\delta \mu^{2}+\frac{D}{2} G(\mu)\right]\langle\phi\rangle^{2} \\
& +\frac{C}{24 \pi}\langle\phi\rangle^{3}+\frac{D}{96 \pi}\langle\phi\rangle^{4} .
\end{aligned}
$$

Substituting next the counterterms (37)-(40) into this result, we have the renormalized version of mean-field energy as function of mean-field value $\langle\phi\rangle$ or $\bar{m}$

$$
\frac{\langle H\rangle}{V}=\frac{1}{8 \pi^{2}}\left[\frac{\pi \mu^{2}}{g^{2}}(m-\bar{m})^{2}+\bar{m}^{4} \ln \left(\frac{\bar{m}}{m}\right)+\frac{\left(\bar{m}^{4}-m^{4}\right)}{4}\right]
$$

where we have added appropriate constant in order to get $\langle H\rangle(\langle\phi\rangle=0)=0$. One can now discuss the possible solution of (42) by analysing the minima of (44).

Let us define $\left.x \equiv g\langle\phi\rangle\right|_{\mathrm{eq}} / m$ and $E(x) \equiv\left(8 \pi^{2} / m^{4}\right)\langle H\rangle / V$. Eqs. (42) and (44) can then be written respectively as 


$$
\frac{\pi \mu^{2}}{2 g^{2} m^{2}} x-(1-x)^{3}\left[\ln (1-x)+\frac{1}{2}\right]=0
$$

and

$$
E(x)=\frac{\pi \mu^{2}}{g^{2} m^{2}} x^{2}+(1-x)^{4}\left[\ln (1-x)+\frac{1}{4}\right]-\frac{1}{4} .
$$

The combination $\frac{4 g^{2} m^{2}}{\pi \mu^{2}}$ has been used as an effective coupling constant in Ref. 115.

The behavior of $E(x)$ is shown in Figs.(1)-(4) for several combination of $\mu / m$ and $g^{2}$. Notice first that this function has the domain at $0 \leq x \leq 1$, which is the physical range for the fermion mass. The point $x=0$ corresponds to $\langle\phi\rangle=0$ or $\bar{m}=m$ and $x=1$ is the case when the effective fermion mass $\bar{m}=0$. Qualitatively, the results indicate that the system always presents a single minimum. Figs.(1)-(2) show $E(x)$ for several values of $g^{2}$ with $\mu / m$ fixed (see figure captions for numerical values of the parameters). The positions of $x_{\text {min }}$ indicate that the vacuum approaches $x=0$ when we decrease $g^{2}$. In the limit of $g^{2} \rightarrow 0$, one gets $x_{\text {min }} \rightarrow 0$, as shown in Fig.(3). In this case, $m \approx \bar{m}$ is the optimal fermion mass, as it must be in the free field theory. On the other hand, Figs.(3)-(4) plot the function $E(x)$ keeping the value of the Yukawa coupling $g^{2}$ fixed, but with different values of the ratio $\mu / m$. Comparing these two curves one sees $x_{\min } \rightarrow 0$ when $\mu / m \rightarrow \infty$. In other words, when the meson mass is large, the force range is small, as usual in the Yukawa theory. In the limit of infinity $\mu$, the fermion particles of the system cannot interact. It can be seen also from the AH's formula, where $m / \mu$ plays roles of effective coupling constant. The above discussions suggest that the field has always a stable vacuum. This means that there is a finite range around the minimum where the dynamics of the system is well defined. In the next section, we shall therefore discuss renormalization conditions for the time-dependent equations.

\section{Renormalization and Initial Conditions for the Time-Dependent Equations}

The last section has discussed the problem of renormalization for the vacuum sector of the 
relativistic scalar plasma model in the Gaussian mean-field approximation. We have shown that the physical quantities can be made finite with the counterterms introduced in (22) and (37)-(40). Here we shall consider an off-equilibrium situation and study renormalizability for the Gaussian equations of motion for this model. (See, e.g., Refs. 16, 17] for the issue of renormalization of time-dependent equations in $\phi^{4}$ field theory)

\section{A. Equations of Motion and Initial State}

In order to pose the problem more clearly, let us begin by rewriting Eq.(24), with the help of $(37)-(40)$, as

$$
\langle\ddot{\phi}\rangle=-\mu^{2}\langle\phi\rangle-8 \pi g \mathcal{F}(t)
$$

where

$$
\mathcal{F}(t)=\int_{\mathrm{k}} \mathcal{F}_{\mathrm{k}}(t)=\int_{\mathrm{k}}\left[\frac{m}{k_{0}} \cos 2 \varphi_{\mathrm{k}}+\frac{k}{k_{0}} \sin 2 \varphi_{\mathrm{k}} \cos \gamma_{\mathrm{k}}+\frac{\bar{m}(t)}{k}-\frac{\bar{m}^{3}(t)}{2 k^{2} k_{0}}\right] .
$$

Notice that the integral is divergent unless $\mathcal{F}_{\mathrm{k}}$ decreases faster than $k^{-3}$. For simplicity, we shall ignore here the possibilities of fractional powers, logarithmic or oscillatory behavior and take the convergence condition for the integral to be

$$
\lim _{k \rightarrow \infty} \mathcal{F}_{\mathrm{k}}=\mathcal{O}\left(\frac{1}{k^{4}}\right)
$$

Thus, the allowed domains for the dynamical variables in momentum space suffer strong constraints, and our investigation of renormalizability of the equations of motion consists in analysing the large momentum behavior of $\mathcal{F}_{\mathrm{k}}$ from this point of view. In order to satisfy (49) at all times the pairing variables $\varphi_{\mathrm{k}}$ and $\gamma_{\mathrm{k}}$ must have their time evolution restricted. These restrictions are analysed better by making the variable changes

$$
k_{0}^{2} \cos 2 \varphi_{\mathrm{k}}=-m R_{\mathrm{k}}-k \sqrt{k_{0}^{2}-R_{\mathrm{k}}^{2}}
$$

or, equivalently,

$$
k_{0}^{2} \sin 2 \varphi_{\mathrm{k}}=-k R_{\mathrm{k}}+m \sqrt{k_{0}^{2}-R_{\mathrm{k}}^{2}}
$$


and

$$
\cos \gamma_{\mathrm{k}}=1-W_{\mathrm{k}} .
$$

In terms of these new variables we have

$$
\mathcal{F}(t)=\int_{\mathrm{k}} \mathcal{F}_{\mathrm{k}}^{(1)}+\int_{\mathrm{k}} \mathcal{F}_{\mathrm{k}}^{(2)}
$$

where

$$
\begin{aligned}
& \mathcal{F}_{\mathrm{k}}^{(1)}=-\frac{R_{\mathrm{k}}}{k_{0}}+\frac{\bar{m}(t)}{k}-\frac{\bar{m}^{3}(t)}{2 k^{2} k_{0}} \\
& \mathcal{F}_{\mathrm{k}}^{(2)}=\frac{k}{k_{0}^{3}} W_{\mathrm{k}}\left(-k R_{\mathrm{k}}+m \sqrt{k_{0}^{2}-R_{\mathrm{k}}^{2}}\right) .
\end{aligned}
$$

We see in $\mathcal{F}_{\mathrm{k}}^{(1)}$ that the leading term of $R_{\mathrm{k} \rightarrow \infty}$ must be $\bar{m}$ in order to cancel the quadratic divergence. Thus, we define

$$
\lim _{k \rightarrow \infty} R_{\mathrm{k}}=\bar{m}+S_{\mathrm{k}}
$$

valid for large $k$. Using this in (54) one gets

$$
\begin{aligned}
\lim _{k \rightarrow \infty} \mathcal{F}_{\mathrm{k}}^{(1)} & =\frac{\bar{m} m^{2}}{k k_{0}\left(k+k_{0}\right)}-\frac{\bar{m}^{3}}{2 k^{2} k_{0}}-\frac{S_{\mathrm{k}}}{k_{0}} \\
& =\frac{1}{k_{0}}\left[\frac{\bar{m}\left(m^{2}-\bar{m}^{2}\right)}{k\left(k+k_{0}\right)}-S_{\mathrm{k}}\right]+\mathcal{O}\left(\frac{1}{k^{5}}\right) .
\end{aligned}
$$

There is still a logarithmic divergence left. To keep this under control we must have

$$
\lim _{k \rightarrow \infty} S_{\mathrm{k}}=\frac{\bar{m}\left(m^{2}-\bar{m}^{2}\right)}{k\left(k+k_{0}\right)}
$$

in order to get finite $\int_{\mathrm{k}} \mathcal{F}_{\mathrm{k}}^{(1)}$.

We examine next the convergence condition for $\int_{\mathrm{k}} \mathcal{F}_{\mathrm{k}}^{(2)}$. Using (56) and (58) in (55) we find the asymptotic expression for $\mathcal{F}_{\mathrm{k}}^{(2)}$

$$
\lim _{k \rightarrow \infty} \mathcal{F}_{\mathrm{k}}^{(2)}=\left[\frac{k^{3}\left(\bar{m}^{2}-m^{2}\right)}{k_{0}^{3}\left(k \bar{m}+k_{0} m\right)}+\mathcal{O}\left(\frac{1}{k^{3}}\right)\right] W_{\mathrm{k}} .
$$


This will constrain the large- $k$ behavior of $W_{\mathrm{k}}$ to be

$$
\lim _{k \rightarrow \infty} W_{\mathrm{k}}=\mathcal{O}\left(\frac{1}{k^{3}}\right)
$$

in order to satisfy (49).

The asymptotic conditions (56), (58) and (60) restrict the dynamics of the Gaussian variables in the moment space. Whenever the Fourier spectrum $\mathcal{F}_{\mathrm{k}}$ has such properties Eqs. (47)-(48) are well defined, and one ought to use these also as a criterion for choosing initial conditions. Since, however, we are dealing with a time dependent problem, the question we want to address to ourselves is whether the nonlinear evolution will distort the asymptotic behavior built into the initial state. From the energy viewpoint, we argue that, if the relations (56), (58) and (60) result a finite energy density, its conservation will enforce these relations at all time. In fact, as we shall show below, the asymptotic behavior obtained in (56), (58) and (60) are suffcient to make (28) finite.

\section{B. Asymptotic Conditions and Energy Density}

In terms of new variables $R_{\mathrm{k}}$ and $W_{\mathrm{k}}$ the energy density (28) can be rewritten as

$$
\frac{\langle H\rangle}{V}=\frac{1}{8 \pi}\left[\langle\Pi\rangle^{2}+\langle\phi\rangle^{2}\right]-2 \int_{\mathrm{k}} \mathcal{E}_{\mathrm{k}}^{(1)}-2 \int_{\mathrm{k}} \mathcal{E}_{\mathrm{k}}^{(2)},
$$

where

$$
\begin{aligned}
& \mathcal{E}_{\mathrm{k}}^{(1)}=\frac{2 k}{k_{0}} \sqrt{k_{0}^{2}-R_{\mathrm{k}}^{2}}+\frac{2 \bar{m} R_{\mathrm{k}}}{k_{0}}-\frac{\bar{m}^{2}}{k}+\frac{\bar{m}^{4}}{4 k^{2} k_{0}}, \\
& \mathcal{E}_{\mathrm{k}}^{(2)}=\frac{(\bar{m}-m) k}{k_{0}^{3}} W_{\mathrm{k}}\left(-k R_{\mathrm{k}}+m \sqrt{k_{0}^{2}-R_{\mathrm{k}}^{2}}\right) .
\end{aligned}
$$

Substracting an unimportant constant $2 k$ from $\mathcal{E}_{\mathrm{k}}^{(1)}$ we find, after a little algebra,

$$
\mathcal{E}_{\mathrm{k}}^{(1)}=-k\left[\frac{R_{\mathrm{k}}}{k_{0}}-\frac{\bar{m}}{k}\right]^{2}+\frac{k}{k_{0}}\left[\frac{\bar{m}^{4}}{4 k^{3}}-\frac{R_{\mathrm{k}}^{4}}{k_{0}\left(k_{0}+\sqrt{k_{0}^{2}-R_{\mathrm{k}}^{2}}\right)^{2}}\right]
$$

from which it follows that

$$
\lim _{k \rightarrow \infty} \mathcal{E}_{\mathrm{k}}^{(1)}=-\frac{k}{k_{0}^{2}}\left(-\frac{\bar{m} m^{2}}{k\left(k+k_{0}\right)}+S_{\mathrm{k}}\right)^{2}+\frac{k}{k_{0}}\left[\frac{\bar{m}^{4}}{4 k^{3}}-\frac{\bar{m}^{4}}{4 k_{0}^{3}}+\mathcal{O}\left(\frac{1}{k^{4}}\right)\right] .
$$


where we have used (56). It is clear now that this expression decrease as fast as $k^{-5}$ and $\int_{\mathrm{k}} \mathcal{E}_{\mathrm{k}}^{(1)}$ is finite. Comparing next (63) with (55) one notes immediately that

$$
\lim _{k \rightarrow \infty} \mathcal{E}_{\mathrm{k}}^{(2)} \sim \lim _{k \rightarrow \infty} \mathcal{F}_{\mathrm{k}}^{(2)}
$$

Hence, $\int_{\mathrm{k}} \mathcal{E}_{\mathrm{k}}^{(2)}$ is finite when the condition $(60)$ is satisfied.

The above qualitative discussion shows that the prescriptions given in (56), (58) and (60) can make energy density finite. They will thus define the initial states and the system will develop a well defined time evolution within a limited moment space. Because of the energy conservation, the asymptotic behavior of the Gaussian variables essentially will not be modified by the dynamics, otherwise this will cost infinite amount of energy from the system. This discussion has not considered, however, possibilities of eventual runaway, caused by eventual unbounded below behavior of the potential. The studies of this problem will require further detailed analysis of $\langle H\rangle$ as multidimensional function of $\bar{m}, R_{\mathrm{k}}$ and $W_{\mathrm{k}}$, (one coordinate for each $k$ ), and verification of its funcional properties [17].

\section{Concluding Remarks}

In summary, we have presented in this paper a framework to treat the initial-value problem for interacting fermion-scalar field models. The method allows one to describe the real-time evolution of the fields in terms of the dynamics of few observables yielding a set of selfconsistent equations for expectation values of linear and bilinear field operators. Although the procedure is quite general, we have however implemented the calculation within the simplest context of relativistic scalar plasma system. We have shown in detail that the usual form of renormalization also applies to the present nonperturbative calculation and we have

obtained finite expression for energy density. A simple numerical calculation suggests that the system has always a single stable minimum, although further investigation will be necessary for other oscillation modes. The standard approach to this question is throught the use of RPA analysis, where the stability is indicated by its the eigenvalues. It is interesting to 
mention here that the excitation modes described by the RPA equations are the quantum particles of the field. In fact, the physics of one meson and two spin-1/2 fermion can be investigated from this equation. We have also discussed the renormalization for the timedependent equations. Using energy conservation as the key, we found that there are a finite range around the vacuum where the dynamics of the system is well defined.

Finally, we comment that systematic corrections to include dynamical correlation effects to the present mean-field calculations can in principle be readily applied with the help of a projection technique discussed in I and Ref. [9]. In this case, the occupation numbers are no longer constant and will affect the effective dynamics of the Gaussian observables. The framework presented here serves also as groundwork to finite density and finite temperature discussions [18]. In particular, a finite-matter density calculation beyond the mean-field approximation allows one to study collisional observables such as transport coefficients [19]. The extension of this procedure to explore nonuniform systems is straighforward but lengthy. In this case, the spatial dependence of the field are expanded in natural orbitals of extented one-body density. These orbitals can be given in terms of a momentum expansion through the use of a more general Bogoliubov transformation [20].

\section{ACKNOWLEDGMENTS}

E.R.T.N. and C-Y. L. are supported by Conselho Nacional de Desenvolvimento Científico e Tecnológico (CNPq), Brazil.

\section{References}

[1] P.M. Stevenson Phys. Rev. D 32, 1389 (1985); P.M. Stevenson and R. Tarrach, Lett. B 176, 436 (1986).

[2] D. Boyanovski, D. Cormier, H. J. de Vega and R. Holman, Phys. Rev. D 55, 3373 (1997).

[3] G-A. Camelia, J. D. Bjorken and S. E. Larsson, Phys. Rev. D 566942 (1997). 
[4] O. Éboli, S.-Y. Pi and R. Jackiw, Phys. Rev. D 37, 3557 (1988); A.K. Kerman and D. Vautherin, Ann. Phys. (N.Y.) 192, 408 (1989).

[5] S.K. Kim, K.S. Soh and J. H. Yee, Phys. Rev. D 411345 (1990).

[6] L.S. Celenza and C.M. Shakin, Phys. Rev. C 24, 2704 (1981).

[7] C-Y. Lin and A. F. R. de Toledo Piza, Mod. Phys. Lett. A 5, 1605 (1990); A.F.R. de Toledo Piza and C-Y. Lin, in Quantum Many-Body Dynamics in the Gaussian Approximation and Beyond, International Conference on Many-Body Nuclear Physics, edited by C. Fiolhais, M. Fiolhais, C. Souza and J. N. Urbano, 277 (World Scientific 1994).

[8] C-Y. Lin and A. F. R. de Toledo Piza, Phys. Rev. D 46, 742 (1992); P. L. Natti and A. F. R. de Toledo Piza, ibid D 54, 7867 (1996). Hereafter these papers are referred to as I and II respectively.

[9] E.R. Takano Natti e A.F.R. de Toledo Piza, Physica A236, 321 (1997); E. R. Takano Natti, Doctoral Thesis, University of São Paulo, 1998 (unpublished).

[10] E.T. Jaynes and F.W. Cummings, Proc. IEEE 51, 89 (1963).

[11] G. Kalman, Phys. Rev. 161, 156 (1967); G. Kalman, Phys. Rev. D 9, 1656 (1974).

[12] C. R. Willis and R. H. Picard, Phys. Rev. A9, 1343 (1974).

[13] M. C. Nemes and A. F. R. de Toledo Piza, Phys. Rev. C 27, 862 (1983).

[14] P.M. Stevenson, G.A. Hajj and J.F. Reed, Phys. Rev. D 34, 3117 (1986).

[15] G Marx, Nucl. Phys. 1, 660 (1956); Ya. B. Zeldovich, Soviet Physics JETP 14, 1143 (1962); E. R. Harrison, Astrophys. J. 142, 1643 (1965); J. D. Walecka, Ann. Phys. 83, 491 (1974); R. Hakim, Riv. N. Cim. 1, 1 (1978); J. D. Alonso and R. Hakim, Phys. Rev. D 29, 2690 (1984).

[16] S.-Y. Pi and M. Samiullah, Phys. Rev. D 36, 3128 (1987); F. Cooper and E. Mottola, Phys. Rev. D 36, 3114 (1987).

[17] A. K. Kerman and C-Y. Lin, Ann. Phys. (N.Y.) 241, 185 (1995).

[18] P. Tommasini and A.F.R. de Toledo Piza, Ann. Phys. (N.Y.) 253, 198 (1997). 
[19] A.F.R. de Toledo Piza, in Proceeding of the IX Workshop in Nuclear Physics, Buenos Aires, Argentina 1986, edited by A.O. Machiavelli, H.M., Sofia, and E. Ventura (World Scientific, Singapore, 1987).

[20] P. Ring and P. Schuck, The Nuclear Many-body Problem, Cap. 7 (Springer-Verlag, New York, 1980).

\section{Figure Captions}

FIGURE 1 . The behavior of the ground-state mean-field energy density $E(x)$ of the scalar plasma system as a function of fermionic effective mass $x=g\langle\phi\rangle / m=1-\bar{m} / m$ for any values of the coupling constant $g$ and mass scale $\mu / m=0.1$ fixed.

FIGURE 2. The behavior of the ground-state mean-field energy density $E(x)$ of the scalar plasma system as a function of fermionic effective mass $x=g\langle\phi\rangle / m=1-\bar{m} / m$ for any values of the coupling constant $g$ and mass scale $\mu / m=2$ fixed.

FIGURE 3. The behavior of the ground-state mean-field energy density $E(x)$ of the scalar plasma system as a function of fermionic effective mass $x=g\langle\phi\rangle / m=1-\bar{m} / m$ for coupling constant $g^{2}=\pi / 100$ and mass scale $\mu / m=2$.

FIGURE 4 . The behavior of the ground-state mean-field energy density $E(x)$ of the scalar plasma system as a function of fermionic effective mass $x=g\langle\phi\rangle / m=1-\bar{m} / m$ for coupling constant $g^{2}=\pi / 100$ and mass scale $\mu / m=0.1$. 